\title{
AN ADAPTIVE FLOATING NODE BASED FORMULATION FOR PROGRESSIVE FATIGUE ANALYSIS OF MULTIPLE DELAMINATIONS
}

\author{
Guillem Gall Trabal $^{1}$, Brian L. V. Bak ${ }^{1}$, Boyang Chen $^{2}$ and Esben Lindgaard $^{1, *}$ \\ ${ }^{1}$ The CraCS Research Group, Aalborg University, Fibigerstræde 16, 9220 Aalborg East, Denmark \\ ${ }^{2}$ Faculty of Aerospace Engineering, Delft University of Technology, Delft, the Netherlands \\ *elo@mp.aau.dk
}

Fatigue-driven delamination is one of the main damage modes leading to the final failure of composite laminated structures. Great advances have been achieved in recent years on accurate modelling of delamination onset and growth under fatigue loading by the use of Cohesive Zone Models (CZM) integrated into cohesive interface finite elements. Although the implementation of such models in Finite Elements Analysis (FEA) through decohesion elements is available in literature and research codes, resulting models are still computationally expensive and require intensive modelling work by the user. These disadvantages make the simulation of multiple delaminations on a general layup at the structural level an impossible task. A new analysis methodology named the Floating Node Method (FNM) has recently been proposed [1] that has the potential to overcome these issues. In this work, a new numerical fatigue formulation based on an FNM enhanced element and a new adaptive refinement scheme is presented. The FNM-based element is capable of including new cohesive elements at any interface in the model as well as refining existing elements without the use of remeshing. The adaptive refinement scheme is based on local information at the element level, i.e. damage state, without hardcoded and problem-dependent user inputs. The suggested adaptive refinement scheme successfully refine and coarse the mesh adaptively as well as, include $\mathrm{CZ}$ elements at needed interfaces during the iterative solution procedure rendering the solution accurate and efficient. The delamination growth in the different interfaces is accounted for using a fatigue model based on [2] which combines a quasistatic cohesive zone model with a Paris law to assess the delamination growth using an envelope approach. The results shown in this presentation showcase the computational cost reduction when using the adaptive FNM based formulation as well as its multiple delamination modelling capabilities.

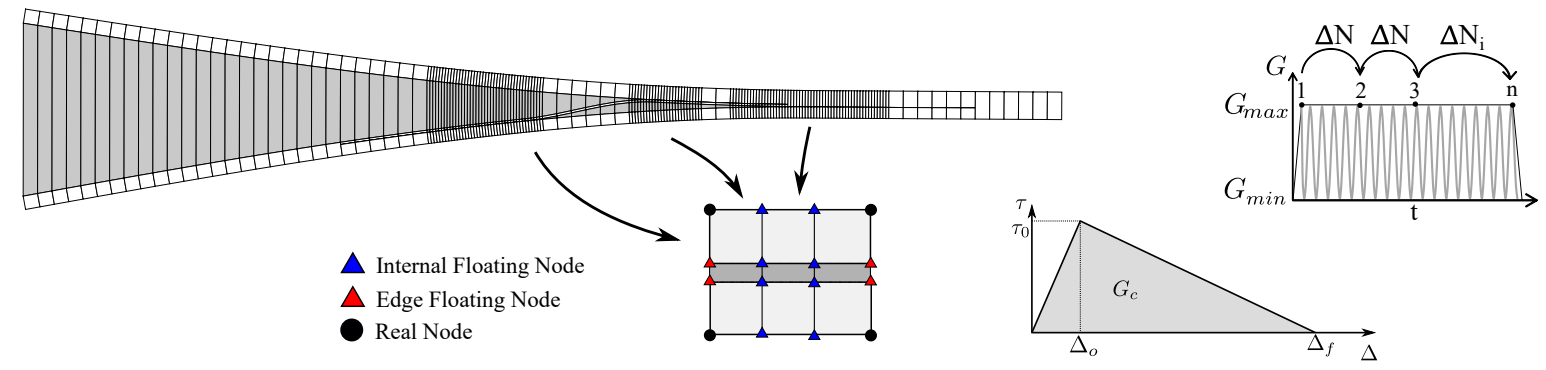

Figure 1: The presented formulation uses local refinement through the FNM, cohesive zone modelling and an envelope fatigue formulation. The result is the efficient simulation of multiple delaminations propagation under fatigue loading.

\section{References}

[1] Chen B., Pinho S. and De Carvalho N. A floating node method for the modelling of discontinuities in composites. Engineering Fracture Mechanics (2014) 127:104-134.

[2] B.L.V Bak., A. Turon., E. Lindgaard and E. Lund, A simulation method for high-cycle fatiguedriven delamination using a cohesive zone model. Int. J. Numer. Meth. Engng (2016) 106:163191. 